\title{
Lifestyle and self-management determinants of hypertension control in a sample of
}

\section{Australian adults}

\author{
Saman Khalesi ${ }^{1}$, Christopher Irwin ${ }^{2}$, Jing Sun $^{3}$ \\ ${ }^{1}$ Physical Activity Research Group, Appleton Institute and School of Health, Medical and \\ Applied Sciences, Central Queensland University, Australia \\ ${ }^{2}$ Menzies Health Institute Queensland and School of Allied Health Sciences, Griffith \\ University, Gold Coast, Australia \\ ${ }^{3}$ Menzies Health Institute Queensland and School of Medicine, Griffith University, Australia \\ Correspondence: Saman Khalesi (Ph.D.). Physical Activity Research Group, Appleton \\ Institute and School of Health, Medical and Applied Sciences, Central Queensland \\ University, Australia. e-mail: s.khalesi@cqu.edu.au P: +61 0432402710
}

ORCID ID: Saman Khalesi 0002-8208-2518; Christopher Irwin 0002-1379-4271; Jing Sun 0002-0097-2438

Author contributions: Khalesi and Sun were involved in the conception, design and analysis of the study. Khalesi collected data. Khalesi, Irwin and Sun were involved in the interpretation of results and preparation of the manuscript.

Acknowledgements: The authors acknowledge the valuable support of the Gold Coast City Council, Gold Coast University Hospital, Griffith University, and all individuals who assisted with data collection, and participated in this study. All authors have read and approved the submission of the manuscript. Authors declare that there is no conflict of interest. Authors declare that there is no source of financial support for this work. 


\section{Abstract}

Background: Poor hypertension control can increase the risk of cardiovascular, renal and kidney diseases, and increase the social and economic burden of the disease. This study aimed to explore the lifestyle and self-management skills (medication adherence, selfmonitoring, self-efficacy) determinants of hypertension control in a sample of hypertensive Australians. Research design and method: In a cross-sectional design, a total of 233 hypertensive adults completed a survey. Hypertension control was categorised as good control if hypertensive individuals managed to reduce their blood pressure (BP) $<140 / 90$ $\mathrm{mmHg}$, otherwise it was categorised as poor control. Data were analysed using bivariate analysis and hierarchical logistic regression. Results: 55\% of the participants had poor BP control. A significantly higher frequency of poor BP self-monitoring was observed in individuals with poor control compared to their counterparts. Poor self-monitoring and a sedentary lifestyle were associated with higher odds of poor control (OR: 5.33, 95\% CI: 1.78 - 15.93; $\mathrm{p}<0.01$, and OR: 4.69, 95\% CI: 1.00 - 22.25; $\mathrm{p}<0.05$, respectively). No significant association was observed between other variables and hypertension control. Conclusions: Successful BP control for hypertensive individuals may require interventions and strategies that assist with improving BP self-monitoring skills and increasing physical activity.

Keywords: Hypertension; Blood pressure control; Lifestyle; Self-management; Selfmonitoring 


\section{Introduction}

The world has an ageing population, partially due to reduced mortality and increased infertility [1]. Yet, while people live longer, ageing can increase the prevalence of chronic disease and bring burden to society, the economy and the health system [1, 2]. The increased need for health care associated with these changes has shifted the health system from considering patients as the passive recipients of care, to actively engaging patients in treating and managing their conditions [3]. However, the success of patients self-management depends on their day-to-day ability to follow lifestyle changes and symptom management strategies provided by health professionals [3]. Successful self-management can result in effective control of the chronic condition [4].

Hypertension (HTN) (characterised by continuous BP $\geq 140 / 90 \mathrm{mmHg}$ ) is a major chronic condition affecting over 3.1 million people in Australia [5]. In 2011/12, 68\% of all Australian adults with HTN did not effectively control their condition [6]. Uncontrolled BP can lead to cardiovascular disease (CVD), renal and kidney failure and increase the social and economic burden of disease [7]. Patients with newly diagnosed hypertension are advised to modify their lifestyle and diet [7]. Individuals with HTN are advised to follow a healthier diet (low in salt, SFA and high in fruit and vegetables), increase their levels of physical activity, reduce alcohol intake and stop smoking [8, 9]. However, adherence to diet and lifestyle modifications is challenging [10,11]. Good knowledge of appropriate diet and lifestyle choices may increase an individual’s confidence to follow a healthy lifestyle [12]. In addition to lifestyle modification, most individuals with HTN need AHT to control their BP [7]. However, adherence to AHT is also quite poor and depends on an individual's confidence and motivation to follow the required treatment [13]. 
In recent years, there has been increasing interest in the importance of self-management skills for treating and controlling HTN [14]. Based on the Individual and Family Self-Management Theory (IFSMT), self-management is fundamental to behavioural change and chronic disease control [15]. In this theory, behavioural change self-efficacy, the ability to self-monitor a condition and its fluctuations, and the adherence to treatment determine the success of selfmanagement [16]. In addition, based on the context dimension of IFSMT, individuals' physical and mental wellbeing or HRQoL [17] can influence their confidence to change their behaviour $[18,19]$. Successful diet and lifestyle modifications may lead to an improvement in health-related quality of life (HRQoL) [20, 21]. Based on the IFSMT outcome dimension, a successful lifestyle modification can also improve behavioural change self-management and self-efficacy [15, 19, 22]. Finally, based on the Knowledge-Attitude-Behaviour (KAB) approach, individuals with a better understanding of dietary factors and their relationship with HTN are more likely to follow a healthy diet and manage their condition effectively [23, 24].

Therefore, the conceptual framework of this study is designed based on the IFSMT theory and is presented in Figure 1. Based on this framework, this study hypothesised that a) there is a difference in nutrition knowledge, dietary patterns, lifestyle, HRQoL and self-management skills of individuals with HTN that have good and poor BP control, and b) these variables are associated with poor BP control in a sample of Australian adults with high BP. Understanding the determinants of poor BP control in individuals with HTN is essential for developing interventions and approaches that aim to improve HTN control among Australian adults. 


\section{Patients and Methods}

\subsection{Design and data collection}

The design and data collection process of this study is described in details elsewhere [25, 26]. Briefly, a clinical- and community-based cross-sectional study collected data between August and December 2014 using a convenience method of sampling. The Clinical unit of sampling was the Medical Assessment Unit of the Gold Coast University Hospital, and the Community units were the Gold Coast City Council Active and Healthy Classes; and Griffith University. The residents of the City of Gold Coast, Australia were also invited to participate, and were included in the Community unit of sampling. Adults ( $\geq 18$ years of age) with high BP were included in this study. High BP or HTN was defined as SBP $\geq 140 \mathrm{mmHg}$ and/or DBP $\geq 90$ mmHg, or medically diagnosed with high BP. In cases where no record of an individual's BP was available, the researcher measured BP in duplicate readings using a validated BP monitor (method explained elsewhere [25]). Those unable to consent to the study, or individuals with severe mental or neurological impairment, or with secondary HTN (due to other causes such as kidney or endocrine problems) were excluded from the study. Ethics approvals were obtained from the Gold Coast University Hospital, and Griffith University ethical committees (HREC Reference Number: HREC/14/QGC/115).

A survey that included information on food frequency, nutrition knowledge, HRQoL, lifestyle, AHT adherence, self-monitoring skills, as well as sociodemographic and anthropometric variables was used to collect data. The food frequency questionnaire (FFQ) developed in Blue Mountains Eye Study [27] was modified and validated for this study and detailed elsewhere [28]. A total of 39 commonly consumed food items, in addition to questions on added salt, oil, and sugar during cooking or serving foods were selected for the modified FFQ. The questionnaire was validated against 3-day food records and had a good 
internal consistency (Cronbach’s alpha $=0.66$ ), with a good Bland-Altman relative agreement [28]. Factor analysis with Varimax rotation was firstly used to identify survey structure, and subsequent cluster analysis was used to identify dietary patterns. The detailed explanation of the procedure and analysis is presented elsewhere [25]. Three patterns of Western, Snack \& Alcohol, and Balanced were derived using cluster analysis. The Western pattern was high is sodium and SFA and loaded with fast-food, processed foods and alcohol. Snack and alcohol pattern was also high in sodium and loaded with chips, crisps, fries, biscuits and alcohol, and the Balanced pattern was a moderate combination of all food groups [25].

The nutrition knowledge questionnaire used in this study was a modified version of the one developed by Hendrie et al. [29] with 26 questions on the dietary recommendation, source of food and nutrients, everyday food choices, and diet-disease relationship. A moderate internal consistency with $\alpha=0.54$ was observed in this newly modified questionnaire. HRQoL was examined by the Short Form 12 (SF-12, V2) [30] with an acceptable internal consistency ( $\alpha=$ 0.66). Physical activity, smoking, over-drinking and stress were examined using short questions. The self-efficacy of diet and exercise behaviour changes were assessed using the shortened versions from the one developed by Sallis et al. [31]. The internal consistency of self-efficacy questionnaires was good with alpha levels of 0.76 and 0.89 , for self-efficacy of diet (with 8 questions) and self-efficacy of exercise (with 4 question), respectively. A fouritem questionnaire developed for this study and described before [26] was used for assessing AHT adherence. Answers were scored on a 3-point scale. Scores 0-2 were considered 'poor adherence', and score 3 was coded as 'good adherence'.

Self-monitoring BP was measured using a 4-item questionnaire, which included statements on: having a BP monitor at home; the frequency of BP measuring; and the individual's feeling of their ability to control BP (2 questions). To score BP self-monitoring, one point was given to having a BP monitor at home and measuring BP at least once a week. Those 
who felt good about the BP control received another point. Those who believed they were able to control their BP with the guidance from their health professional received another point. No point was given to the remaining options. BP self-monitoring was categorised as good (score 4) and poor (score 0-3). The BP self-monitoring questionnaire had an acceptable internal consistency with $\alpha=0.66$ [32]. Blood pressure control was defined as a good control if individuals had controlled their BP (SBP $<140 \mathrm{mmHg}$ and DBP $<90 \mathrm{mmHg}$ ). Blood pressure control was checked against the average of BP readings and was confirmed with individuals and the medical professional team (if applicable).

\subsection{Statistical analysis}

The process of data cleaning and management is described elsewhere [25]. The bivariate analyses of variables were conducted using chi-square for categorical variables and independent t-tests for normally distributed outcome variables, or Mann-Whitney U tests for non-normally distributed outcome variables.

Based on the conceptual framework (Figure 1) and the IFSMT theory, the association between dietary patterns, nutrition knowledge, lifestyle, HRQoL and BP self-management skills with BP control was explored using a hierarchical regression analysis [33]. Four models were identified. In model 1, the association of nutrition knowledge alone and poor BP control was explored. In model 2, the association of dietary patterns and lifestyle variables (physical activity, alcohol overconsumption and smoking) in addition to nutrition knowledge with poor BP control was investigated. In model 3, HRQoL was added to the regression model and its association with poor BP control was assessed. In model 4 the self-management skills (selfmonitoring, AHT adherence, self-efficacy of diet and exercise) variables were added to nutrition knowledge, dietary pattern, lifestyle and HRQoL and their association with poor BP 
control were assessed. All models were controlled for the confounding influence of significant bivariate sociodemographic variables and sampling units. The stress frequency variable was not included in the regression analysis as it had a significant correlation with the HRQoL total mental domain $(0.37, \mathrm{p}<0.01)$. P-value $<0.05$ was considered as a statistically significant level.

The sample size was calculated based on previous work by Bramley, Nightengale [34], where 43\% of individuals with good AHT adherence successfully achieved BP control compared to 32\% in low adherence individuals. Therefore, using GPower software version 3.0 (Heinrich Heine Universitat, Dusseldorf, Germany), with $80 \%$ power and $\alpha=0.05$, a sample size of 177 was required to examine associations in this study. Including an additional $20 \%$ for noncompleted or missing survey responses, a sample size of 213 was considered adequate.

\section{Results}

A total of 233 hypertensive adults were included. More than half (55\%) of these individuals had poor BP control. Of these 224 reported currently taking AHT medication to control BP. Table 1 presents the characteristics of included participants allocated to the poor and good BP control groups. The cross-tabulation analysis of sociodemographic factors shows a significant ethnicity difference between poor and good BP control, with more Caucasians in the good control compared to the poor control group. However, a small group of individuals were included in the group of other ethnicities with good control, which may have influenced the overall results. A significantly higher proportion of individuals with health and nutritionrelated qualifications was observed among adults with poor BP control compared to those with good control. No significant differences were observed among other variables. 
Table 2 presents differences between the poor and good BP control groups in terms of AHT types and numbers, their comorbidities and sampling units. A significant difference was observed with the sampling units, with the majority of individuals in the good BP control group being from the Clinical sampling unit.

Table 3 presents differences between the poor and good BP control groups with respect to nutrition knowledge, dietary patterns, HRQoL, self-efficacy, lifestyle, BP self-monitoring and AHT adherence. A significant difference was observed in BP self-monitoring between groups with good and poor BP control. Only 9\% of individuals with poor BP control had good BP self-monitoring, however, a higher proportion (22\%) of those with good BP self-monitoring had good BP control. No significant differences were observed in nutrition knowledge, dietary patterns and HRQoL between the good and poor BP control groups.

The association between nutrition knowledge, dietary patterns, lifestyle factors, HRQoL, BP self-management skills and poor BP control is presented in Table 4. Except for model 1, all models had good fit based on the Hosmer-Lemeshow test. In model 1, nutrition knowledge alone was not a significant determinant of BP control while controlling for confounding variables of ethnicity, health-related qualifications and sampling units. Dietary patterns and lifestyle variables (physical activity, smoking, alcohol overconsumption) were added to model 2. A higher likelihood of having poor BP control was observed when paired with a sedentary lifestyle compared to a rigorously active lifestyle. A similar pattern was detected when HRQoL total physical and total mental domains were added to model 3. In model 4, self-management skills (self-monitoring, AHT adherence and self-efficacy) were included. Poor BP self-monitoring increased the odds of poor BP control more than 5 times. In this model, sedentary lifestyle also increased the likelihood of poor BP control by more than 4.5 times. All models were controlled for ethnicity, health-related qualification and sampling 
units. Being Caucasian reduced the odds of poor BP control in all models. No confounding influence of health-related qualification or sampling units was observed on the association.

\section{Discussion}

In the present study, over half of the study sample did not have their BP under good control. Similar rates of poor BP control among individuals with HTN has also been identified in the US population [35]. Findings from the present study suggest that individuals with poor BP control have lower self-management skills than individuals with good BP control. Based on IFSMT theory [15], success with BP control at the individual level is largely related to an individual's self-management skills and their involvement in the treatment and control of their condition [2, 3]. Hypertensive individuals, especially in the early stages, usually do not experience physical symptoms, therefore, may not feel the need to adhere to treatment or control of their condition [36]. However, regular BP self-monitoring may increase awareness of the condition, the need for action and an individual's active involvement in treatment [37]. Based on IFSMT, self-involvement in the treatment of a condition is a pivotal step in selfmanaging and controlling that condition [15]. The associations observed in this study support the importance of BP self-monitoring for the control of HTN. In addition, the results show that good control of BP was more common in individuals from the clinical setting. This may be due to frequent BP monitoring that occurs in clinical settings and constant updating of treatment plans to improve the control of HTN and other comorbidities. However, regression analysis in this study did not suggest a significant confounding influence of the setting on HTN control. Also, results indicated that good BP control was more common in individuals with no health-related qualifications. This finding may have been influenced by the low number of participants with health-related qualification included in this study. Although the 
confounding influence of health-related qualifications on regression models of BP control was not significant, further investigation is required to explore potential reasons for differences observed.

The findings of this study also reported lower involvement in physical activity for individuals with poor BP control compared to those with good BP control. As designated in the outcome dimension of IFSMT theory, an individual's motivation to change and to self-manage their condition is very much influenced by having successful lifestyle modification outcomes [15]. Therefore, having an inactive lifestyle may limit the success of BP self-management, and reduce the likelihood of BP control. This was further supported by the findings of the present study where associations between physical inactivity and lower likelihood of BP control was observed. Sedentary lifestyles have long been considered a major culprit in many chronic diseases, including HTN, obesity and diabetes [10]. The importance of physical activity to reduce BP has been well investigated in epidemiological and experimental studies [38, 39, 40]. A number of mechanisms have been proposed for this effect. Reducing the circulating catecholamines in serum, decreasing plasma noradrenaline levels and renin activity, and reducing the peripheral vascular resistance are among these [38, 39].

In the present study, no differences in nutrition knowledge, dietary patterns, HRQoL, smoking, overconsumption of alcohol or adherence to medication between groups with poor or good BP control was observed. Although the conceptual framework of this study suggested that these variables may increase the likelihood of BP self-management and control, the findings of this study did not report such associations. Nevertheless, the importance of diet and lifestyle modifications in targeting HTN treatment and control is undeniable [7]. There is compelling evidence suggesting diet plays a critical role in the prevention or onset of HTN [7, 41]. There is no doubt that Western dietary patterns high in salt (sodium), SFA, alcohol and low in fruit and vegetables increases the risk of HTN [7, 41]. 
Individuals are more likely to choose healthier foods when they have better knowledge of dietary factors [42]. There is also overwhelming evidence for the association between smoking and adverse cardiovascular outcomes [11, 43]. Heavy alcohol consumption has also been associated with higher BP in previous reports [44, 45]. Although these associations were not observed in the current study, it is possible that a combination of these factors, along with other behavioural factors (such as belief, accessibility and availability of food and resources), may be associated with HTN control, since it is a multifactorial condition [7].

To the best of our knowledge, the current study is one of few to have explored associations between nutrition knowledge, dietary patterns, lifestyle factors, HRQoL, BP selfmanagement skills and BP control in Australian adults with HTN. Overall, the study suggests that a sedentary lifestyle and poor BP self-monitoring can reduce the likelihood of successful BP control.

While the findings of this study have valuable public health and clinical implications, there are several limitations that should be noted. The small population sample of the study makes the generalisation of findings difficult. However, participants from both Clinical and Community units were included in the study to increase the generalisation of the results. Also, to partial out the sampling unit differences, this study controlled the confounding influence of sampling units in the analyses. Another limitation of this study is that the majority of the population were Caucasians, which limited the comparison and generalisation of results to other ethnicities. In addition, data collection in this study was conducted using a short self-administered questionnaire, which can introduce reporting bias [46]. For the same reason, some missing and incomplete information (i.e. AHT medication type and name and comorbidities) existed that limited the analysis and interpretation of results. With regards to alcohol consumption, only overconsumption of alcohol (> 2 standard drinks per day for men, or $>1$ standard drink per day for women, or $>4$ standard drinks in a single drinking occasion) 
was included in this study. Therefore, participants were not able to report actual levels of alcohol consumed. In addition, the smoking frequency question was not able to identify the exact number of cigarettes smoked each day or the number of years an individual had been smoking for. Also, the length of diagnosis may influence the success of BP control. However, accurate diagnosis duration recall from individuals with a long history of diagnosis was limited. Due to the limited access this study had to the recorded medical history, especially from individuals in the Community sampling unit, this information was not included. Finally, BP control was defined and classified based on average BP readings and confirmed with medical professionals. However, BP readings are volatile can fluctuate for a number of reasons including the state of other comorbidities, stress and day-to-day variations. Thus, it is important to consider this when interpreting the findings. Future intervention based studies focusing on a cause-effect relationship between these variables and BP control are required to confirm the findings of this study.

\section{Conclusion}

Overall, the findings of this study emphasise the importance of BP self-monitoring and physical activity in BP control for adults with HTN. Accordingly, hypertensive individuals with poor BP self-monitoring and those following a sedentary lifestyle have lower likelihood of achieving successful BP control. These findings may provide a strategic direction for clinical and public health interventions focusing on HTN control. Educating hypertensive adults to enhance BP self-measurement skills, implementing policies for greater accessibility and affordability of BP monitoring devices, improving knowledge of BP control and increasing individuals’ self-control confidence may lead to better BP self-management skills, thus, better BP control. This, along with strategies to improve diet and lifestyle, may 
effectively improve rates of BP control among hypertensive individuals, reduce treatment costs and both the clinical and financial burden associated with the disease. 


\section{References}

1. World Population Aging [Internet]. New York Department of Economic and Social Affairs, Population Division, United Nations. 2013. Available from: http://www.un.org/en/development/desa/population/publications/pdf/ageing/WorldPo pulationAgeing2013.pdf

2. Bodenheimer T, Lorig K, Holman H, et al. Patient self-management of chronic disease in primary care. JAMA: The Journal of the American Medical Association. 2002;288:2469-75

** In this landmark article self-management and self-efficacy are presented as important determinants to control and manage chronic disease, highlighting the need for education to improve self-management in patients.

3. Barlow J, Wright C, Sheasby J, et al. Self-management approaches for people with chronic conditions: A review. Patient Educ Couns. 2002;48:177-87

4. Norris SL, Engelgau MM, Narayan KV. Effectiveness of self-management training in type 2 diabetes: A systematic review of randomized controlled trials. Diabetes Care. 2001;24:561-87

* This study supports the effectiveness of self-management education and training in managing type 2 diabetes.

5. Australian Health Survey: Health Service Usage and Health Related Actions, 2011-12 [Internet]. 2013. Available from:

http://www.abs.gov.au/ausstats/abs@.nsf/lookup/322DB1B539ACCC6CCA257B390 $\underline{\text { 00F316C?opendocument }}$

6. High blood pressure statistics [Internet]. Heart Fundation Australia. 2012. Available from: http://www.heartfoundation.org.au/SiteCollectionDocuments/Factsheet-Highblood-pressure.pdf 
7. Chobanian AV, Bakris GL, Black HR, et al. Seventh report of the joint national committee on prevention, detection, evaluation, and treatment of high blood pressure. Hypertension. 2003;42:1206-52

\section{** This remarkable article provides a guideline for HTN prevention and treatment and important factors influencing HTN control including the role of patients.}

8. Escott-Stump S, Krause MV, Mahan LK, et al. Krause's food \& the nutrition care process. St. Louis, Mo: Elsevier/Saunders; 2012. (Book, Whole)

9. Appel LJ, Brands MW, Daniels SR, et al. Dietary approaches to prevent and treat hypertension: A scientific statement from the American Heart Association. Hypertension. 2006;47:296-308 doi: 10.1161/01.hyp.0000202568.01167.b6

10. Tremblay MS, Colley RC, Saunders TJ, et al. Physiological and health implications of a sedentary lifestyle. Appl Physiol Nutr Metab. 2010;35:725-40

11. Huang J, Huang B, Chen Z, et al. Analysis of the prevalence and risk factors of hypertension in the She population in Fujian, China. Kidney \& blood pressure research. 2011;34:69-74 doi: 10.1159/000323164

12. Heaney S, O'Connor H, Michael S, et al. Nutrition knowledge in athletes: A systematic review. International Journal of Sport Nutrition and Exercise Metabolism. 2011 Jun;21:248-61 PubMed PMID: 21719906 eng

13. Nelson M, Reid C, Ryan P, et al. Self-reported adherence with medication and cardiovascular disease outcomes in the Second Australian National Blood Pressure Study (ANBP2). Med J Aust. 2006;185:487-9

14. McManus RJ, Mant J, Bray EP, et al. Telemonitoring and self-management in the control of hypertension (TASMINH2): A randomised controlled trial. The Lancet. 2010;376:163-72 
15. Ryan P, Sawin KJ. The individual and family self-management theory: Background and perspectives on context, process, and outcomes. Nurs Outlook. 2009;57:217-25. e6

16. Bandura A. Social foundations of thought and action: A social cognitive theory. USA: Prentice-Hall, Inc; 1986.

17. Suurmeijer TP, Reuvekamp MF, Aldenkamp BP. Social functioning, psychological functioning, and quality of life in epilepsy. Epilepsia. 2001;42:1160-8

18. Koenigsberg MR, Bartlett D, Cramer JS. Facilitating treatment adherence with lifestyle changes in diabetes. Am Fam Physician. 2004;69:309-24

19. Amarantos E, Martinez A, Dwyer J. Nutrition and quality of life in older adults. The Journals of Gerontology Series A, Biological Sciences and Medical Sciences. 2001;56 Spec No 2:54-64 doi: 10.1093/gerona/56.suppl_2.54

20. Penedo FJ, Dahn JR. Exercise and well-being: A review of mental and physical health benefits associated with physical activity. Current Opinion In Psychiatry. 2005;18:189-93

21. Kuczmarski MF, Sees AC, Hotchkiss L, et al. Higher Healthy Eating Index-2005 scores associated with reduced symptoms of depression in an urban population: Findings from the Healthy Aging in Neighborhoods of Diversity Across the Life Span (HANDLS) study. J Am Diet Assoc. 2010;110:383-9

22. Drewnowski A, Evans WJ. Nutrition, physical activity, and quality of life in older adults summary. The Journals of Gerontology Series A: Biological Sciences and Medical Sciences. 2001;56:89-94

23. van der Wal MH, Jaarsma T, Moser DK, et al. Compliance in heart failure patients: The importance of knowledge and beliefs. Eur Heart J. 2006;27:434-40 
24. Contento IR. Nutrition education: Linking research, theory, and practice. Asia Pacific Journal of Clinical Nutrition 2008;17:176-9

25. Khalesi S, Sharma S, Irwin C, et al. Dietary patterns, nutrition knowledge and lifestyle: associations with blood pressure in a sample of Australian adults (the Food BP study). J Hum Hypertens. 2016 Apr 28 doi: 10.1038/jhh.2016.22 PubMed PMID: 27121443 Eng

* This study highlighted the importance of dietary patterns and other lifestyle behaviours in the development of HTN in a sample of Australian adults.

26. Khalesi S, Irwin C, Sun J. Dietary Patterns, Nutrition Knowledge, Lifestyle, and Health-Related Quality of Life: Associations with Anti-Hypertension Medication Adherence in a Sample of Australian Adults [journal article]. High Blood Pressure \& Cardiovascular Prevention. 2017 September 07 doi: 10.1007/s40292-017-0229-9

** This is an important article suggesting adherence to HTN medication may reduce if patients have poor lifestyle and self-efficacy to follow a healthier lifestyle.

27. Smith W, Mitchell P, Reay EM, et al. Validity and reproducibility of a selfadministered food frequency questionnaire in older people. Aust N Z J Public Health. 1998 Jun;22:456-63 PubMed PMID: 9659773 eng

28. Khalesi S, Doshi D, Buys N, et al. Validation of a short food frequency questionnaire in Australian adults. International Journal of Food Sciences and Nutrition. 2016:1-9 doi: 10.1080/09637486.2016.1240763

29. Hendrie GA, Coveney J, Cox D. Exploring nutrition knowledge and the demographic variation in knowledge levels in an Australian community sample. Public Health Nutrition. 2008;11:1365-71 doi: 10.1017/s1368980008003042 
30. Mancia G, Sega R, Bombelli M, et al. Metabolic syndrome in the Pressioni Arteriose Monitorate E Loro Associazioni (PAMELA) study: Daily life blood pressure, cardiac damage, and prognosis. Hypertension. 2007;49:40-7 doi:

10.1161/01.hyp.0000251933.22091.24

31. Sallis JF, Pinski RB, Grossman RM, et al. The development of self-efficacy scales for healthrelated diet and exercise behaviors. Health Education Research. 1988;3:283-92

32. Turconi G, Celsa M, Rezzani C, et al. Reliability of a dietary questionnaire on food habits, eating behaviour and nutritional knowledge of adolescents. Eur J Clin Nutr. 2003;57:753-63

33. Victora CG, Huttly SR, Fuchs SC, et al. The role of conceptual frameworks in epidemiological analysis: A hierarchical approach. Int J Epidemiol. 1997;26:224-7

34. Bramley TJ, Nightengale BS, Frech-Tamas F, et al. Relationship of blood pressure control to adherence with antihypertensive monotherapy in 13 managed care organizations. Journal of Managed Care Pharmacy. 2006;12:239-45

35. Yoon SSB, V.; Louis,T.; Carrol M.D. Hypertension among adults in the United States, 2009-2010. NCHS data brief. 2012;107:1-8

36. Gascón JJ, Sánchez-Ortuño M, Llor B, et al. Why hypertensive patients do not comply with the treatment: Results from a qualitative study. Fam Pract. 2004;21:12530

\section{* This study shows the importance of understanding patient's priorities and education in the management of HTN.}

37. Cappuccio FP, Kerry SM, Forbes L, et al. Blood pressure control by home monitoring: Meta-analysis of randomised trials. BMJ: British Medical Journal. 2004;329:145 doi: 10.1136/bmj.38121.684410.AE 
38. Zortéa K, Tartari RF. Arterial hypertension and physical activity. Arq Bras Cardiol. 2009;93:414-5

39. Fagard RH. Exercise is good for your blood pressure: Effects of endurance training and resistance training. Clin Exp Pharmacol Physiol. 2006 Sep;33:853-6 doi: 10.1111/j.1440-1681.2006.04453.x PubMed PMID: 16922820 eng

40. Fagard RH. Exercise characteristics and the blood pressure response to dynamic physical training. Med Sci Sports Exerc. 2001;33:S484-S92

41. Aburto NJ, Ziolkovska A, Hooper L, et al. Effect of lower sodium intake on health: Systematic review and meta-analyses. Br Med J. 2013;346:f1326 doi: 10.1136/bmj.f1326 PubMed PMID: 23558163 eng

42. Zoellner J, Connell C, Bounds W, et al. Nutrition literacy status and preferred nutrition communication channels among adults in the Lower Mississippi Delta. Preventing Chronic Disease. 2009 Oct;6:A128 PubMed PMID: 19755004 PubMed Central PMCID: PMCPMC2774642. eng

43. Ambrose JA, Barua RS. The pathophysiology of cigarette smoking and cardiovascular disease: An update. J Am Coll Cardiol. 2004;43:1731-7

44. Briasoulis A, Agarwal V, Messerli FH. Alcohol consumption and the risk of hypertension in men and women: A systematic review and meta-analysis. The Journal of Clinical Hypertension. 2012;14:792-8

45. Nagao T, Hase T, Tokimitsu I. A green tea extract high in catechins reduces body fat and cardiovascular risks in humans. Obesity. 2007;15:1473-83 doi: 10.1038/oby.2007.176

46. Burns KE, Duffett M, Kho ME, et al. A guide for the design and conduct of selfadministered surveys of clinicians. Can Med Assoc J. 2008;179:245-52 


\section{Key issues:}

- Poor hypertension control is common in hypertensive patients which can lead to the development of cardiovascular disease and renal and kidney problems.

- Lifestyle changes and self-management (i.e. self-efficacy, medication adherence, selfmonitoring) are proposed as means to improve hypertension management and control.

- This study suggests poor blood pressure self-monitoring and sedentary lifestyle can be significant determinants of poor hypertension control.

- Improving hypertensive patient's self-monitoring skills and physical activity may be effective approaches to improve hypertension control.

- Future interventional studies need to confirm these findings. 
1 Table 1 Characteristics of included participants in good and poor BP control

\begin{tabular}{|c|c|c|c|}
\hline & $\begin{array}{l}\text { Poor control, n } \\
(\%)(n=130)\end{array}$ & $\begin{array}{l}\text { Good control, } n \\
(\%)(n=103)\end{array}$ & $\chi^{2}$ (p-value) \\
\hline Number & $130(55)$ & $103(45)$ & \\
\hline Age (years of age) & & & $1.08(0.29)$ \\
\hline$<65$ & $77(59)$ & $54(52)$ & \\
\hline$\geq 65$ & $53(41)$ & $49(48)$ & \\
\hline Gender & & & $1.21(0.27)$ \\
\hline Male & $50(38.5)$ & $47(46.0)$ & \\
\hline Female & $80(61.5)$ & $56(54.0)$ & \\
\hline Ethnicity & & & $6.87(0.00)$ \\
\hline Caucasian & $114(89.0)$ & $98(98.0)$ & \\
\hline Other & $14(11.0)$ & $2(2.0)$ & \\
\hline Marital status & & & $0.34(0.84)$ \\
\hline Married, de facto & $82(63.0)$ & $67(66.0)$ & \\
\hline Divorced, widowed, separated & $38(29.0)$ & $29(28.0)$ & \\
\hline Never been married & $10(8.0)$ & $6(6.0)$ & \\
\hline Education & & & $0.28(0.86)$ \\
\hline Elementary, high school & $65(50.0)$ & $55(53.5)$ & \\
\hline College/ University degree & $48(37.0)$ & $36(35.0)$ & \\
\hline Postgraduate master or $\mathrm{PhD}$ & $17(13.0)$ & $12(12.0)$ & \\
\hline Income & & & $2.38(0.30)$ \\
\hline Less than $20 \mathrm{k}$ & $69(53.0)$ & $63(62.5)$ & \\
\hline $20-<60 k$ & $36(28.0)$ & $20(20.0)$ & \\
\hline$\geq 60 \mathrm{k}$ & $25(19.0)$ & $18(18.0)$ & \\
\hline
\end{tabular}




\begin{tabular}{|c|c|c|c|}
\hline Employment & & & $2.46(0.48)$ \\
\hline Full-time & $37(28.5)$ & $23(22.5)$ & \\
\hline Part-time & $21(16.0)$ & $13(13.0)$ & \\
\hline Unemployed or student & $15(11.5)$ & $11(11.0)$ & \\
\hline Retired & $57(44.0)$ & $55(54.0)$ & \\
\hline Number of people in household & & & $3.30(0.19)$ \\
\hline Single person & $24(19.0)$ & $25(25.0)$ & \\
\hline Medium size (2-4 people) & $89(71.0)$ & $72(71.0)$ & \\
\hline Large size ( $\geq 5$ people) & $12(10.0)$ & $4(4.0)$ & \\
\hline Health-related qualification & & & $4.60(0.03)$ \\
\hline Yes & $35(27.5)$ & $16(16.0)$ & \\
\hline No & $92(72.5)$ & $88(84.0)$ & \\
\hline BMI $\left(\mathrm{kg} / \mathrm{m}^{2}\right)$ & $28.73(6.30)$ & $29.14(6.01)$ & $-0.41(0.64)$ \\
\hline
\end{tabular}

$2 *$ BP is presented as mean (SD). The rest of variables are presented as frequency (\%). Chi-

3 square was used to compare bivariate differences. 
4 Table 2 Medication types, comorbidities and sampling unit differences among participants

5 with good and poor BP control

\begin{tabular}{|c|c|c|c|}
\hline & $\begin{array}{l}\text { Poor control, } n \\
(\%)(n=130)\end{array}$ & $\begin{array}{l}\text { Good control, } n \\
(\%)(n=103)\end{array}$ & $\chi^{2}$ (p-value) \\
\hline Types of AHT & & & $1.21(0.55)$ \\
\hline One type & $65(66)$ & $65(72)$ & \\
\hline Two types & $21(21)$ & $18(20)$ & \\
\hline > Two types & $12(13)$ & $7(8)$ & \\
\hline \multicolumn{4}{|l|}{ Common AHT } \\
\hline ACE inhibitors & $13(15)$ & $12(15)$ & $0.04(0.99)$ \\
\hline Diuretics & $15(18)$ & $15(18)$ & \\
\hline Calcium channel, Beta-blockers or & $12(14)$ & $11(13)$ & \\
\hline \multicolumn{4}{|l|}{ Alpha- receptor blockers } \\
\hline Others, or did not know & $45(53)$ & $44(54)$ & \\
\hline Comorbidities & & & $6.30(0.10)$ \\
\hline Heart problems & $7(14)$ & $13(24)$ & \\
\hline Obesity & $32(62)$ & $22(40)$ & \\
\hline Diabetes/high cholesterol/or Gout & $4(8)$ & $10(18)$ & \\
\hline Multiple comorbidities & $8(16)$ & $10(18)$ & \\
\hline Sampling Unit & & & $10.62(0.00)$ \\
\hline Clinical & $50(39)$ & $58(56)$ & \\
\hline Community & $72(55)$ & $35(34)$ & \\
\hline Active classes & $8(6)$ & $10(10)$ & \\
\hline
\end{tabular}


7 Table 3 Difference in dietary pattern, nutrition knowledge, HRQoL, lifestyle and self-monitoring skills, between groups with good and poor BP 8 control

\begin{tabular}{|c|c|c|c|}
\hline & Poor control, n (\%) (n=130) & $\begin{array}{l}\text { Good control, } \mathrm{n}(\%) \\
(\mathrm{n}=103)\end{array}$ & t or $\chi^{2}$ (p-value) \\
\hline Nutrition knowledge score (out of 100) & $65.90(13.71)$ & $64.00(13.60)$ & $1.10(0.27)$ \\
\hline Dietary Pattern & & & $2.31(0.31)$ \\
\hline Western & $54(42.0)$ & $35(33.5)$ & \\
\hline Snack \& Alcohol & $49(37.5)$ & $40(39.0)$ & \\
\hline Balanced & $27(20.5)$ & $28(27.5)$ & \\
\hline \multicolumn{4}{|l|}{ HRQoL } \\
\hline Physical function & $65.70(35.50)$ & $63.48(38.16)$ & $0.45(0.64)$ \\
\hline Role physical & $66.47(30.89)$ & 61.76 (34.59) & $1.09(0.27)$ \\
\hline Bodily pain & $35.66(32.74)$ & $44.12(38.08)$ & $-1.78(0.07)$ \\
\hline General health & $54.07(24.95)$ & $48.53(26.05)$ & $1.64(0.10)$ \\
\hline Total Physical & $55.47(15.53)$ & $54.47(18.90)$ & $0.44(0.66)$ \\
\hline Vitality & 47.09 (21.79) & 48.77 (24.97) & $-0.54(0.58)$ \\
\hline
\end{tabular}




\begin{tabular}{|c|c|c|c|}
\hline Social function & $70.16(30.47)$ & $69.85(29.08)$ & $0.07(0.93)$ \\
\hline Role emotional & $74.42(25.13)$ & $72.92(29.17)$ & $0.42(0.67)$ \\
\hline Mental health & 57.95 (13.79) & $59.19(18.52)$ & $-0.58(0.56)$ \\
\hline Total Mental & $62.40(14.12)$ & $62.68(15.36)$ & $-0.14(0.88)$ \\
\hline \multicolumn{4}{|l|}{ ifestyle variables } \\
\hline \multicolumn{4}{|c|}{ Physical activity (hour/week) } \\
\hline Vigorous & $1.90(3.85)$ & $2.68(4.60)$ & $-1.33(0.18)$ \\
\hline Moderate & $6.60(7.49)$ & $8.22(8.88)$ & $-1.47(0.14)$ \\
\hline Light & $21.90(21.10)$ & $19.46(20.59)$ & $0.87(0.38)$ \\
\hline Sitting activity & $29.16(19.15)$ & $29.02(18.07)$ & $0.05(0.95)$ \\
\hline Sleeping & $47.42(15.69)$ & $48.07(16.80)$ & $-0.29(0.77)$ \\
\hline \multicolumn{4}{|c|}{ Physical activity categories ${ }^{1}$} \\
\hline Sedentary & $45(36.0)$ & $26(26.0)$ & $3.01(0.22)$ \\
\hline Moderate & $69(54.5)$ & $61(60.0)$ & \\
\hline Rigorous & $12(9.5)$ & $14(14.0)$ & \\
\hline
\end{tabular}

Smoking, n (\%) 
No

Used to

Yes

Over drinking, n (\%)

Never

Often

Sometimes

Used to

Stress, n (\%)

Never

Often

Sometimes

Occasionally

Self-management Skills

BP self-monitoring

Poor
78 (64.5)

30 (25.0)

13 (10.5)

56 (46.0)

13 (11.0)

34 (27.5)

19 (15.5)

17 (14.0)

27 (22.0)

45 (37.0)

33 (27.0)

47 (47.0)

$11(11.0)$

34 (34.0)

$8(8.0)$

17 (16.0)

$26(26.0)$

$29(29.0)$

$28(28.0)$

$1.90(0.38)$

$3.28(0.35)$

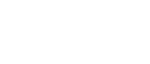


Good

Self-efficacy

Diet (out of 100)

Exercise (out of 100)

AHT adherence

Poor

Good
$12(9)$

70.91 (17.69)

$63.62(23.80)$

50 (48)
$-0.82(0.40)$

$-1.25(0.21)$

$9 \quad{ }^{1}$ Moderate: physical activities for at least 30 minutes, 5 times per week, which does not cause heavy breathing (e.g. walking, hiking, gardening); Rigorous:

10 physical activities for at least 20 minutes, 3 time per week, which cause sweating and heavy breathing (e.g. running, football, shovelling).

11 Data presented as mean (SD) or frequency (\%). Independent sample t-test and chi-squared test are used were appropriate. Significant p-value level is $<0.05$.

12 Abbreviations: AHT: anti-hypertensive medication; HRQoL: health-related quality of life 
Table 4 The association between nutrition knowledge, dietary pattern, Lifestyle, HRQoL, BP self-management skills, with poor BP control

\begin{tabular}{|c|c|c|c|c|}
\hline Poor BP control & Model 1 & Model 2 & Model 3 & Model 4 \\
\hline \multicolumn{5}{|l|}{ Dietary pattern } \\
\hline Western (vs Balance) & - & $1.37(0.61-3.07)$ & $1.43(0.63-3.22)$ & $0.81(0.29-2.24)$ \\
\hline Snack \& Alcohol (vs Balance) & - & $1.26(0.58-2.71)$ & $1.23(0.57-2.67)$ & $0.73(0.28-1.91)$ \\
\hline \multicolumn{5}{|l|}{ Lifestyle variables } \\
\hline \multicolumn{5}{|l|}{ Physical activity categories } \\
\hline Sedentary (vs Rigorous) & - & $3.15(1.10-9.03)^{*}$ & $3.12(1.04-9.30)^{*}$ & $4.69(1.00-22.25)^{*}$ \\
\hline Used to (vs No) & - & $2.19(0.64-7.50)$ & $2.03(0.58-7.08)$ & $4.15(0.75-22.83)$ \\
\hline Yes (vs No) & - & $0.89(0.45-1.76)$ & $0.87(0.43-1.72)$ & $0.64(0.26-1.58)$ \\
\hline \multicolumn{5}{|l|}{ Over drinking, n (\%) } \\
\hline Often (vs Never) & - & $0.77(0.28-2.08)$ & $0.84(0.29-2.37)$ & $0.42(0.09-2.02)$ \\
\hline
\end{tabular}


Sometimes (vs Never)

Used to (vs Never)

HRQoL

Total physical

Total mental

Self-management skills

BP self-monitoring, Poor (vs Good) -

AHT adherence, Poor (vs Good)

Self-efficacy

Diet

\section{Exercise}

Ethnicity, Caucasian (vs Others)

Health-related qualification, No (vs Yes)

Sampling unit

Clinical (vs Active classes)

Community (vs Active classes)

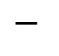

$-$

$-$
$0.97(0.48-1.92)$

$2.27(0.79-6.49)$

$-$

$-$

$-$

\begin{abstract}
$0.15(0.97-1.02) *$
$0.16(0.29-0.81)^{*}$
\end{abstract}

$0.63(0.29-1.37)$

$0.78(0.38-1.55)$

$1.23(0.42-3.61)$

$2.42(0.84-6.93)$

$1.34(0.40-4.49)$

$2.13(0.65-6.97)$
$0.98(0.49-1.95)$

$2.25(0.77-6.53)$

$0.90(0.38-2.11)$

$2.60(0.73-9.21)$

$1.00(0.98-1.02)$

$0.98(0.96-1.01)$

$0.99(0.97-1.02)$

$1.00(0.97-1.03)$

$5.33(1.78-15.93)^{* *}$

$0.80(0.35-1.81)$

$0.98(0.95-1.00)$

$1.00(0.98-1.03)$

$0.17(0.03-0.86)^{*}$

$0.09(0.10-0.93)^{*}$

$0.59(0.26-1.30) \quad 1.00(0.38-2.61)$

$1.28(0.38-4.36)$

$2.27(0.50-10.21)$

$2.07(0.62-6.83)$

$4.04(0.91-18.04)$ 


$$
\mathrm{R}^{2}=0.10^{* *} \quad \mathrm{R}^{2}=0.16^{*} \quad \mathrm{R}^{2}=0.16^{*} \quad \mathrm{R}^{2}=0.28^{* * *}
$$

$14{ }^{*}$ p-value $<0.05 ; * *$ p-value $<0.01 ; * * *$ p-value $<0.001$. Good fit models, except for model 1. Model 1 (Nutrition knowledge); Model 2 (Nutrition 15 knowledge + Dietary patterns and sleep duration); Model 3 (Nutrition knowledge + Dietary patterns and sleep duration+ HRQoL); Model 4

16 (Nutrition knowledge + Dietary patterns and sleep duration+ HRQoL + Self-efficacy). All models are controlled for sociodemographic variables

17 (age, gender, employment, and income), and sampling units. Abbreviations: HRQoL: health-related quality of life 


\section{Figure Caption}

19 Figure 1. The conceptual framework of the association between dietary pattern, nutrition

20 knowledge, lifestyle and HRQoL with HTN control 